\title{
IDH mutation-induced suppression of type-1 anti-glioma immune response
}

\author{
Gary Kohanbash ${ }^{*}$, Shruti Shrivastav ${ }^{1}$ Brian Ahn², Yafei Hou', Joseph Costello ${ }^{3}$, Hideho Okada ${ }^{1}$ \\ From 30th Annual Meeting and Associated Programs of the Society for Immunotherapy of Cancer (SITC 2015) \\ National Harbor, MD, USA. 4-8 November 2015
}

Isocitrate dehydrogenase (IDH) mutations are the first mutations that occur during the oncogenic process of lower-grade glioma (LGG) and confers a novel gain-offunction activity by converting $\alpha$-ketoglutarate $(\alpha K G)$ to 2-hydroxyglutarate (2HG), promoting DNA hypermethylation. Our analysis of LGG cases from The Cancer Genome Atlas (TCGA) database revealed that IDHmutant (IDH-Mut) cases exhibit decreased expression of type-1 effector $\mathrm{T}$ cell response-related genes, which are critical for anti-glioma immunity, including: $C D 8 A$, IFNG, OAS2, GZMA, EOMES, CXCL9 and CXCL10, compared with IDH-wild type (IDH-WT) cases. On the other hand, type- 2 and regulatory $\mathrm{T}$ cell responserelated genes, such as $I L 5$ and TGFB1, are not significantly different between IDH-Mut vs. WT cases, indicating that the observed down-regulation of type-1 response-related genes does not merely represent a possible global gene suppression. Furthermore, IDH-Mut cases exhibit increased CXCL1O promotor methylation compared with WT cases. We thus hypothesized that IDH mutation-mediated tumor intrinsic mechanisms occurring within glioma cells may inhibit anti-tumor immunity to promote tumor growth. In vitro, a normal human astrocyte (NHA) cell line transfected with IDH1Mut cDNA expressed lower levels of CXCL10 compared to NHA cells transfected with WT IDH1. Consistently, $\mathrm{C} 57 \mathrm{Bl} / 6$ mouse-syngeneic astrocyte and glioma cell lines transfected with IDH1-Mut expressed lower levels of CXCL10 gene and protein, compared to control cells transfected with IDH-WT, which was restored following 30 day treatment of the cells with the IDH1 inhibitor, IDH-C35. Furthermore, in vivo orthotopic IDH1-Mut gliomas at 21 days post-intracranial injection in syngeneic mice expressed lower levels of $\mathrm{T}$ cell chemokines CXCL9 and CXCL10 as determined by RT-PCR and
ELISA and reduced infiltration of CD3+CD8+ T cells as determined by flow cytometry and quantitative immunohistochemistry compared with control IDH1-WT gliomas. Further, an in vitro migration assay demonstrated reduced migration of $\mathrm{T}$ cells towards culture supernatants from IDH1-Mut cell lines compared with control supernatants derived from IDH1-WT cells. Overall, our data demonstrate that IDH mutations in tumor cells lead to reduced $T$ cell attracting chemokines and reduced $T$ cell accumulation in gliomas. Our analyses of the TCGA $450 \mathrm{~K}$ gene methylation database suggest that the suppressed expression of OAS2 and CXCL1O in IDH1-Mut cases is associated with hypermethylation of the promoter for these genes. Indeed, treatment of IDH-Mut cell lines with demethylating agent 5-Aza-CdR restored CXCL10 expression levels. Our data suggest that IDH inhibitors and demethylation agents may be used to enhance $\mathrm{T}$ cell recruitment to LGG in combination with $\mathrm{T}$ cell based immunotherapies.

\section{Authors' details \\ ${ }^{1}$ University of California, San Francisco, San Francisco, CA, USA. ${ }^{2}$ University of Pittsburgh, Pittsburgh, PA, USA. ${ }^{3}$ University of California San Francisco, San} Francisco, CA, USA.

Published: 4 November 2015

doi:10.1186/2051-1426-3-S2-P271

Cite this article as: Kohanbash et al:: IDH mutation-induced suppression of type-1 anti-glioma immune response. Journal for ImmunoTherapy of Cancer 2015 3(Suppl 2):P271. 